EGU21-56

https://doi.org/10.5194/egusphere-egu21-56

EGU General Assembly 2021

(c) Author(s) 2021. This work is distributed under

the Creative Commons Attribution 4.0 License.

\title{
Statistical Modeling of Non-Stationary Heatwave Hazard
}

\author{
Peng Zhong ${ }^{1}$, Raphael Huser ${ }^{1}$, and Thomas Opitz ${ }^{2}$ \\ ${ }^{1}$ KAUST, Thuwal, Saudi Arabia (raphael.huser@kaust.edu.sa) \\ ${ }^{2}$ INRAE, Avignon, France (thomas.opitz@inrae.fr)
}

The modeling of spatio-temporal trends in temperature extremes can help better understand the structure and frequency of heatwaves in a changing climate, as well as their environmental, societal, economic and global health-related risks. Here, we study annual temperature maxima over Southern Europe using a century-spanning dataset observed at 44 monitoring stations. Extending the spectral representation of max-stable processes, our modeling framework relies on a novel construction of max-infinitely divisible processes, which include covariates to capture spatio-temporal non-stationarities. Our new model keeps a popular max-stable process on the boundary of the parameter space, while flexibly capturing weakening extremal dependence at increasing quantile levels and asymptotic independence. It clearly outperforms natural alternative models. Results show that the spatial extent of heatwaves is smaller for more severe events at higher altitudes and that recent heatwaves are moderately wider. Our probabilistic assessment of the 2019 annual maxima confirms the severity of the 2019 heatwaves both spatially and at individual sites, especially when compared to climatic conditions prevailing in 1950-1975. Our applied results may be exploited in practice to understand the spatio-temporal dynamics, severity, and frequency of extreme heatwaves, and design suitable regional mitigation measures. 